\title{
Lymphocytic Adrenal Medullitis: Case Report of A Patient with Pheochromocytoma-Like Hypertensive Paroxysms
}

\section{(๑) $\circledast \Theta$}

\author{
Authors \\ Jan Calissendorff1, 2, Carl Christofer Juhlin 3, 4
}

\section{Affiliations}

1 Department of Endocrinology, Metabolism and Diabetes, Karolinska University Hospital, Stockholm, Sweden.

2 Department of Molecular Medicine and Surgery, Karolinska Institutet, Stockholm, Sweden

3 Department of Clinical Pathology and Cytology, Karolinska University Hospital, Stockholm, Sweden

4 Department of Oncology-Pathology, Karolinska Institutet, Stockholm, Sweden

Key words

Adrenal, medullitis, inflammation, hypertension

received $\quad 08.08 .2017$

first decision $\quad 18.10 .2017$

accepted $\quad 30.10 .2017$

Bibliography

DOI https://doi.org/10.1055/s-0043-122492

Exp Clin Endocrinol Diabetes Rep 2018; 6: e1-e5

(C) J. A. Barth Verlag in Georg Thieme Verlag KG Stuttgart .

New York

ISSN 2196-7407

Correspondence

Dr C. Christofer Juhlin

Department of Clinical Pathology and Cytology

Karolinska University Hospital

17176, Stockholm

Sweden

Tel.: + 46/8/51770 000

christofer.juhlin@ki.se

\begin{abstract}
Lymphocytic adrenal medullitis (LAM) denote the histological finding of lymphocytic infiltrates in the adrenal medulla, a rare phenomenon that previously has been deemed asymptomatic and linked to autopsy findings in patients with type I diabetes. We describe the finding of LAM in a 66-year old female presenting with hyperaldosteronism and a $17 \mathrm{~mm}$ lesion in the right adrenal gland. The patient displayed secondary hypertension as well as intermittently occurring exaggerated hypertensive episodes with systolic peaks above $220 \mathrm{~mm} \mathrm{Hg}$. Preoperative catecholamine screening was negative, diminishing the possibility of a pheochromocytoma. Unilateral adrenalectomy was performed, and histology revealed an aldosterone producing adrenocortical adenoma. No signs of adrenomedullary hyperplasia or pheochromocytoma were found; however, prominent B- and T-lymphocytic infiltrates were observed across the medulla. The inflammation was almost absent in the surrounding adrenocortical tissue. Postoperatively, the patient remained hypertensive, but without paroxysmal excessive blood pressure elevations. A review of our pathology records between the years 2000 to present time as well as focused histopathological re-examination of 40 cases revealed no signs of LAM in any adrenal gland investigated, confirming the occurrence in living patients as exceedingly rare. This is the first report of LAM in a living patient, with a possible clinical association to intermittent episodes of markedly increased blood pressure not commonly seen in patients with hyperaldosteronism. A coupling between LAM and exaggerated hypertensive episodes cannot be ruled out.
\end{abstract}

\section{Case Presentation}

The patient is a 66-year old female of Caucasian ethnicity with no family history of adrenal disease. She underwent splenectomy in 2005 due to immune thrombocytopenic purpura (ITP) and was admitted to a local hospital in 2009 because of nautical vertigo and hypertension $(220 / 120 \mathrm{mmHg})$. At this point, potassium was normal and she was treated with $10 \mathrm{mg}$ enalapril and $10 \mathrm{mg}$ felodipin. Blood test showed ordinary aldosterone (376 pmol/L) and a low renin (1.2 ng/L), giving an ARR quotient of 313, see $>$ Table 1. $\mathrm{U}$-cortisol was slightly elevated, but there were no signs of Cushing's syndrome. Urine sample of catecholamine was normal. Blood pressure normalized $(135 / 75 \mathrm{~mm} \mathrm{Hg})$ upon treatment. She was also treated with simvastatin since her cholesterol levels were elevated. During the years, her blood pressure was reasonably stable with an addition of $25 \mathrm{mg}$ metoprolol in 2010. 
- Table 1 Laboratory measures at investigations during 2009 and 2016, and at post-operative follow-up 2017.

\begin{tabular}{|l|c|c|c|c|c|}
\hline & $\mathbf{2 0 0 9}$ & $\mathbf{2 0 1 6}$ & $\mathbf{2 0 1 6}$ & $\mathbf{2 0 1 7}$ & \multicolumn{1}{|c|}{ Ref } \\
\hline Potassium & 3.8 & 3.0 & 3.9 & 4.1 & $3.5-4.4 \mathrm{nmol} / \mathrm{L}$ \\
\hline P-aldosterone & 376 & & 474 & 119 & $190-830 \mathrm{pmol} / \mathrm{L}(\mathrm{standing})$ \\
\hline P-renin & 1.2 & & 1.2 & 16 & $3-33 \mathrm{ng} / \mathrm{L}(\mathrm{standing})$ \\
\hline U-aldosterone & & & 35 & & $3.3-78 \mathrm{nmol} / 24 \mathrm{~h}$ \\
\hline ARR & 313 & & 395 & & $>100$ suspect PA \\
\hline U-adrenaline & 36 & & & & $2-80 \mathrm{nmol} / 24$ \\
\hline U-noradrenaline & 313 & & & & $60-400 \mathrm{nmol} / 24 \mathrm{~h}$ \\
\hline fP-metoxyA & & $<0.2$ & $<0.2$ & & $<0.3 \mathrm{nmol} / \mathrm{L}$ \\
\hline fP-metoxyNA & 0.3 & 0.3 & 0.6 & & $<1.1 \mathrm{nmol} / \mathrm{L}$ \\
\hline P-adrenaline & & & $<0.3$ & & $0.7 \mathrm{nmol} / \mathrm{L}$ \\
\hline P-noradrenaline & & 4 & & $1.6-4.8 \mathrm{nmol} / \mathrm{L}(\mathrm{standing})$ \\
\hline U-5HIIA & 26 & & 15 & & $10-80 \mathrm{umol} / \mathrm{L}$ \\
\hline Chromogranin A & & & & & $<3 \mathrm{nmol} / \mathrm{L}$ \\
\hline U-cortisol & & & 593 & & $240-730 \mathrm{~mol} / \mathrm{L}$ \\
\hline S-cortisol & & & & & $<50 \mathrm{nmol} / \mathrm{L}$ \\
\hline S-cortisol after & & & & & \\
\hline dexamethason & & & & \\
\hline P-ACTH & & & & \\
\hline
\end{tabular}

In the fall of 2016, the patient presented with increased and fluctuating blood pressure and later also hypokalaemia, ranging from $3.0-3.3 \mathrm{nmol} / \mathrm{L}$. She was once again admitted at another local hospital because of chest pain, tiredness and occasional hypertensive episodes. Her blood pressure could within $3 \mathrm{~h}$ change from 140/95 in to 270/135. During a three-day stay, paroxysmic elevations in blood pressure were observed. $750 \mathrm{mg}$ of kaleorid normalized potassium levels in doses $5+5$ daily. An increased ARR quotient was once again found. P-metoxynoradrenaline and p-metoxyadrenline was analyzed twice; both normal, as was P-adrenaline and P-noradrenaline. A subsequent CT scan demonstrated a $17 \times 13 \times 10 \mathrm{~mm}$ large mass in the right adrenal with Houndsfield units -3 . Her medication was changed to losartan $100 \mathrm{mg}$, amlodipin $10 \mathrm{mg}$ and doxacozin $4 \mathrm{mg}$ twice daily, which normalized blood pressure (144/62 to $110 / 60 \mathrm{mmHg}$ ). Catheterization of the adrenal veins lateralized to the right. Laparoscopic adrenalectomy was performed at the Karolinska University Hospital to where she was referred. Postoperatively, blood pressure was regulated with $25 \mathrm{mg}$ of metoprolol and $5 \mathrm{mg}$ amlodipine, and her rapid fluctuations in blood pressure vanished after surgery.

\section{Histopathology}

The right adrenal gland weighted 13,2 grams and measured $5 \times 4 \times 1,5 \mathrm{~cm}$. A single $1,5 \times 1,5 \times 1,2 \mathrm{~cm}$ lesion with a yellowish cut surface was observed expanding the adreno-cortex, no further lesions were observed. In addition, the adrenal medulla was focally observed with a white-yellowish cut surface, which is not normally seen when grossing the adrenal gland. Routine histopathology verified the $1,5 \mathrm{~cm}$ lesion as an adrenocortical adenoma composed of a circumscribed lipid-rich cells with a low nuclear-to-cytoplasmic ratio ( $\triangleright$ Fig. 1a). A Weiss score, an established way of determining malignant potential in adrenocortical lesions, yielded zero points, thereby ruling out this lesion as malignant. The lesion was uniformly positive for CYP11B2 (aldosterone synthase) and negative for CYP11B1 (responsible for conversion of progesterone to cortisol) immunoreactivity, verifying the lesion as aldosterone producing ( $\triangleright$ Fig. 1b). The surrounding adrenocortex showed no nodular expansions, thereby excluding micronodular hyperplasia. Moreover, the surrounding tissue showed microscopic cell clusters with intense CYP11B2 immunoreactivity denoted "aldosterone producing cell clusters" (APCC) which are regularly observed in a large subset of non-tumorous adrenals. The clinical significance of APCC is unknown, but recent molecular studies suggest that APCC might be a precursor of aldosterone producing adenomas [1].

The adrenal medulla was focally seen with a slight increase in width ( $3 \mathrm{~mm}$ ), but with no nodular expansions suggestive for adrenomedullary hyperplasia. Moreover, the adrenal medulla was observed with small nodules of mature lymphocytes, but without medullar atrophy or fibrosis ( $\vee$ Fig. 2 a). Immunohistochemical investigations confirmed the nodules as lymphocytic (CD45+), with a predominance of type B-lymphocytes $(C D 20+)$ admixed with $\mathrm{T}_{\text {Helper }}(\mathrm{CD} 4+)$ and $\mathrm{T}_{\text {Killer }}(\mathrm{CD} 8+$ )-lymphocytes ( $\mathbf{F i g}$. 2b). Extensive review of our pathology records revealed no signs of LAM in any adrenal gland $(n=704)$ investigated between the years 2000 to present time, confirming the occurrence of LAM as exceedingly rare in our patient material. To avoid reporting bias, we also reviewed a total of 40 adrenal glands exhibiting non-medullary conditions, for eventual LAM involvement. The cohort's consisted of 21 aldosterone-producing adrenocortical adenomas, 14 adrenocortical hyperplasias (10 aldosterone-producing, 2 cortisol-producing and one with both aldosterone and cortisol production), 2 cortisol-producing adrenocortical adenomas, one non-producing adrenocortical adenoma, one adrenal with metastasing rectal adenocarcinoma and one normal adrenal adjacent to an abdominal paraganglioma. For all these cases, the adrenal medulla was histo- 
a

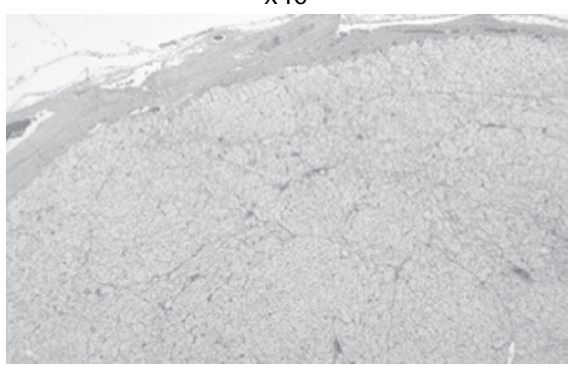

b

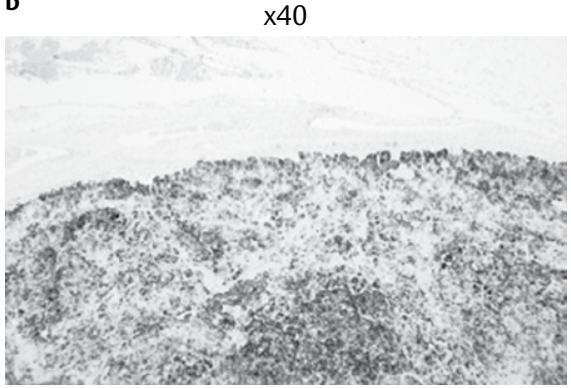

x400

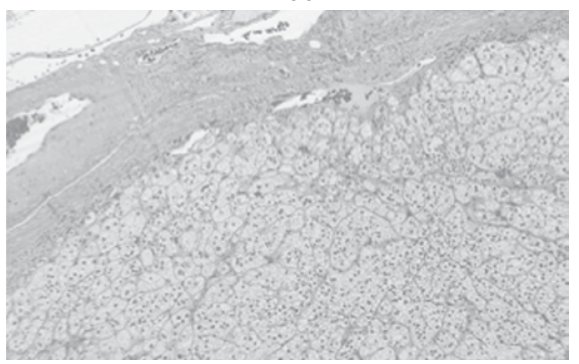

$\mathrm{x} 400$

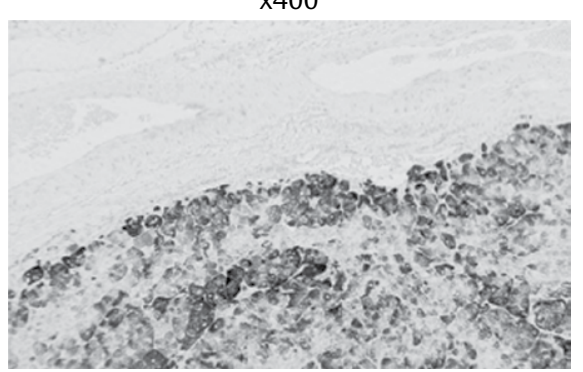

- Fig. 1 a Photomicrographs of a hematoxylin-eosin stained adrenocortical adenoma at $x 40$ and $x 100$ magnifications without vascular or capsular invasion and devoid of prominent inflammation. b CYP11B2 (aldosterone synthase) immunohistochemistry of the adrenocortical adenoma at x40 and x100 magnifications displaying strong and diffuse cytoplasmic immunoreactivity in the majority of tumor cells, while the surrounding capsule and blood vessels stained negative as internal negative controls.

a

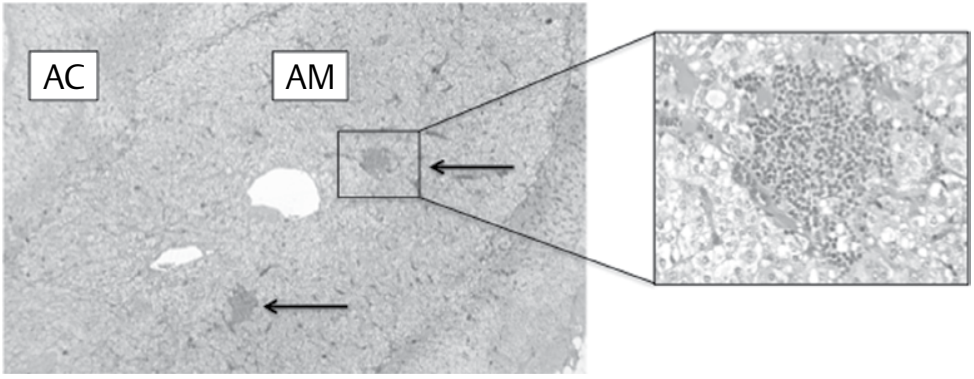

b

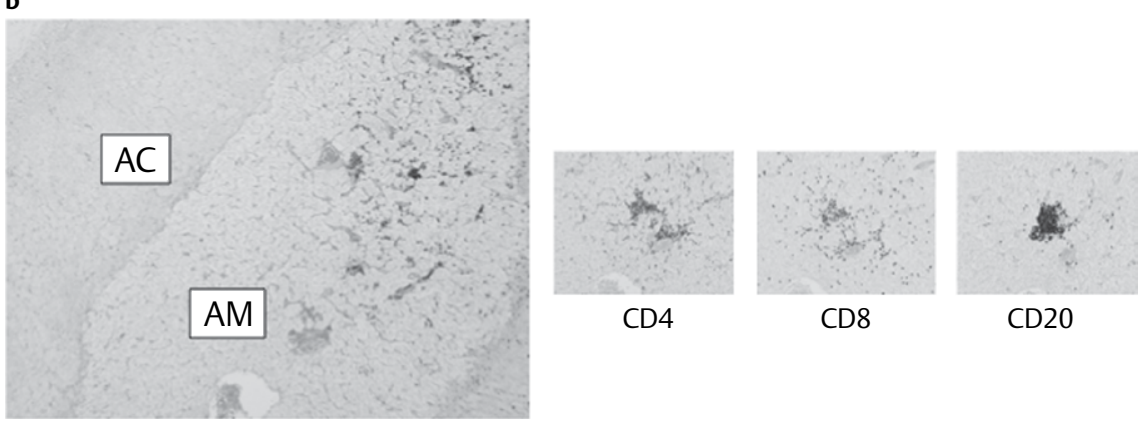

- Fig. 2 a Photomicrograph of hematoxylin-eosin stained adreno-cortex (AC) and adrenal medulla (AM) at x40 magnification, with dense lymphocytic infiltrates (arrows) specifically confined to the latter compartment consistent with adrenal medullitis. Insert is magnified x400. $\mathbf{b}$ Immunohistochemical stainings for the common lymphocytic marker CD45 (large image), as well as CD4 (T helper cells), CD8 (T killer cells) and CD20 (B cells) at x40 magnification. 
logically normal and devoid of nodular lymphocytic infiltrates. This makes us assume that the prevalence of LAM in cases with concurrent adrenocortical disease is low.

\section{Informed Consent}

Informed consent was obtained from the individual participant included in the study.

\section{Discussion}

Lymphocytic adrenal medullitis (LAM) is a rare entity, not least demonstrated by the general absence of scientific articles on the subject. In a retrospective autopsy study in patients with type I diabetes, histological signs of LAM was seen in $20 \%$ of diabetic patients as compared to $3 \%$ of non-diabetic controls [2]. The authors speculate that the adrenal medulla can be a second immunological target in addition to the beta cells of the pancreas. However, a subsequent study performed in non-obese diabetic mice could not detect LAM in the adrenals of any mice with overt diabetes [3]. To add to this, a recent report found evidence of LAM in a dog that also displayed lymphocytic thyroiditis, underscoring the possible co-occurrence between LAM and autoimmune diseases in endocrine organs [4]. Bissel et al. observed LAM in an autopsy report from two twins deceased from Coxsackievirus B4 meningioencefalitis, implying a viral etiology [5]. Others have found evidence of LAM in three out of five patients deceased with rabies, thereby adding evidence to the neurotropic viral theory - not least interesting given the embryological and functional relationships between the adrenal medulla and the nervous system [6].

The coupling between hyperaldosteronism and inflammation is interesting, as mineralocorticoids can mediate inflammatory processes in a variety of organs. Aldosterone promotes inflammation via the mineralocorticoid receptor (MR) and can increase nicotinamide dinucleotide phosphate oxidase levels with secondary oxidative stress in tissues expressing MR [7]. Other inflammatory markers also increase with formation of reactive oxygen species and induction of proinflammatory genes [8]. Also, aldosterone can induce inflammation in cells lacking MR by the enzyme 11- $\beta$-hydroxysteroid dehydrogenase type 2 (11ßHSD-2) [9]. However, MR expression or presence of $11 \beta \mathrm{HSD}-2$ in the adrenal medulla has not been demonstrated. Therefore, other pathways have most likely mediated the inflammation in our patient.

Our patient had been treated for immune thrombocytopenia (ITP), an autoimmune disease characterized by insufficient platelet production and increased destruction resulting in a low platelet count. The mechanism triggering the onset is unknown, but antiplatelet antibodies and T-cell activation contribute. 12 years after splenectomy, the patient exhibits normal platelet counts and no signs of residual ITP. Thus, a direct ITP associated inflammatory effect in the adrenal is unlikely. If other indirect mechanisms, including underlying genetics or existing autoantibodies could mediate medullitis remains speculative.
To our knowledge, this is the first report of LAM in human tissue not acquired from autopsy material, thereby excluding the possibility of post mortal lymphocytic infiltration. Moreover, this is the first case of LAM with a possible association to the clinical presentation. We speculate that the adrenomedullary inflammation perturbed the chromaffin cells and caused intermittent increases in plasma cathecholamine levels, much like the hyperthyroid period seen in the initial phases of a giant cell thyroiditis associated with follicular cell lysis. However, as we did not observe an increase in plasma catecholamines and no standard collection of urine was performed, the assumed coupling between the finding of LAM and the patient's symptomatology remain speculative. Interestingly, the patient is still hypertensive, albeit without the paroxysmal attacks of sudden rise in blood pressure. However, cure of hypertension after adrenalectomy is only noted in two thirds of patients and correlates positively with duration of disease and with the age of the patient [10]. Interestingly, the observed finding of APCC could potentially indicate a predisposition to develop aldosterone-producing lesions also in the contralateral adrenal gland; however the patient is currently displaying normal aldosterone-to-renin ratios, which argue against this theory.

In summary, we report the first case of LAM in a living patient with known autoimmune disease and intermittent sharp increases in systolic blood pressure.

\section{Acknowledgements}

Carl Christofer Juhlin is a Junior Clinical Investigator (Swedish Cancer Society). The study was also funded in by grants provided by the Swedish Society for Medical Research (SSMF).

\section{Conflict of Interest}

The author reports no conflicts of interest in this work.

\section{References}

[1] Nishimoto K, Tomlins SA, Kuick R et al. Aldosterone-stimulating somatic gene mutations are common in normal adrenal glands. Proc Natl Acad Sci USA 2015; 112: E4591-E4599

[2] Brown FM, Smith AM, Longway S et al. Adrenal medullitis in type I diabetes. J Clin Endocrinol Metab. 1990; 71: 1491-1495

[3] Breidert M, Böttner A, Möller S et al. Apoptosis in the adrenal gland of non-obese diabetic (NOD) mice. Exp Clin Endocrinol Diabetes. 1998; 106: 478-483

[4] Doi T, Tomonari Y, Kawasako K et al. Lymphocytic adrenal medullitis and lymphocytic thyroiditis in a laboratory beagle dog. J Vet Med Sci. 2017; 79: 255-257

[5] Bissel S], Winkler CC, DelTondo J et al. Coxsackievirus B4 myocarditis and meningoencephalitis in newborn twins. Neuropathology 2014; 34: 429-437

[6] Almeida Hde O, Teixeira Vde P, de Oliveira G et al. Adrenal medullitis in cases of human rabies. Mem Inst Oswaldo Cruz. 1986; 81: 439-442 
[7] Keidar S, Kaplan M, Pavlotzky E et al. Aldosterone administration to mice stimulates macrophage NADPH oxidase and increases atherosclerosis development: a possible role for angiotensin-converting enzyme and the receptors for angiotensin II and aldosterone. Circulation 2004; 109: 2213-2220

[8] Leroy V, De Seigneux S, Agassiz V et al. Aldosterone activates NF-kappa $B$ in the collecting duct. J Am Soc Nephrol. 2009; 20: 131-144
[9] Qin W, Rudolph AE, Bond BR et al. Transgenic model of aldosteronedriven cardiac hypertrophy and heart failure. Circ Res. 2003; 93: 69-76

[10] Lumachi F, Ermani M, Basso SM et al. Long-term results of adrenalectomy in patients with aldosterone-producing adenomas: multivariate analysis of factors affecting unresolved hypertension and review of the literature. Am Surg. 2005; 71: 864-869 